\title{
Lower Bounds for the Complexity of the Graph of the Hausdorff Distance as a Function of Transformation*
}

\author{
W. J. Rucklidge \\ Xerox Palo Alto Research Center, 3333 Coyote Hill Road. \\ Palo Alto, CA 94304, USA \\ rucklidge@parc.xerox.com
}

\begin{abstract}
The Hausdorff distance is a measure defined between two sets in some metric space. This paper investigates how the Hausdorff distance changes as one set is transformed by some transf ormation group. Algorithms, to find the minimum distance as one set is transformed have buen described, but few lower bounds are known. We consider the complexity of the graph of the Hausdorff distance as a function of transformation, and exhibit some constructions that give lower bounds for this complexity. We exhibit lower-bound constructions for both sets of points in the plane, and sets of points and line segments; we consider the graph of the directed Hausdorff distance under translation, rigid motion, translation and scaling, and affine transformation. Many of the results can also be extended to the undirected Hausdorff distance. These lower bounds are for the complexity of the graph of the Hausdorff distance, and thus do not necessarily bound algorithms that search this graph; however, they do give an indication of how complex the search may be.
\end{abstract}

\section{Introduciuun}

The Hausdorff distance betwecin two sets $A$ and $B$ is defined as

$$
H(A, B)==\max (h(A, B), h(B, A)),
$$

where

$$
h(A, B)=\sup _{a \in A} \inf _{b \in B}\|a-b\|
$$

and $\|\cdot\|$ is some norm (here restricted to be some $L_{p}$ norm). This paper examines the

* This work was supported in part ty National Science Foundation PYI Grant IRI-9057928 and matching funds from General Electric, Kodak, and Xerox, and in par by Air Force Contract AFOSR-91-0328. 
case where $A$ and $B$ are compact subsets of the plane consisting of either a finite number of points, or a finite number of points and nonintersecting line segments.

$h(A, B)$ is called the directed Hausdorff distance from the set $A$ to the set $B . H(A, B)$ is the undirected Hausdorff distance between the sets $A$ and $B . h(A, B)$ is small exactly when every point in $A$ is close to some point in $B ; h(B, A)$ is small when every point in $B$ is close to some point in $A$, and $H(A, B)$ is small when both of these are true. In particular, $h(B, A) \leq \varepsilon$ exactly when for any $b \in B$ there is some $a \in A$ such that $\|a-b\| \leq \varepsilon$. Let $A^{\varepsilon}=A \oplus D(\varepsilon)$ where $\oplus$ is the Minkowski sum, and $D(\varepsilon)$ is the closed disk of radius $\varepsilon$ (the set of all points $x$ such that $\|x\| \leq \varepsilon$ ). A key observation [1]. [4] is that, for compact sets such as we are considering, $h(B, A) \leq \varepsilon$ iff $B \subseteq A^{\varepsilon}$.

In many problems, we want to determine the transformation of one set which brings it into closest correspondence with the other set. Let $G$ be some group of transformations. Then for any $g \in G$ define

$$
F_{G}(g)=H(A, g(B)) \text {. }
$$

In other words, we transform the set $B$ by some transformation $g$ and compute the Hausdorff distance between this transformed set and $A$. This defines a function of $g$, and we wish to determine the minimum value of this function, as the transformation which gives rise to this minimum value is the one bringing $B$ into closest correspondence with $A$. Many approaches to determining this minimizing transformation are based on searching the graph of this function (for example, by enumerating the local minima, as in [5]). It is therefore of interest to know what the geometric complexity of this graph may be. Upper bounds have been determined for some transformation groups. but few lower bounds were known [5], [4]. We also consider the graph of the function

$$
f_{G}(g)=h(g(B), A)
$$

which is the graph of the directed distance from the transformed set $g(B)$ to $A$.

We exhibit lower bounds for the complexity of such graphs as follows. Each construction is parametrized by two values, $\varepsilon$ and $n$ (and possibly other parameters). For each one, we fix some values for $\varepsilon, n$, and any other parameters, and construct sets $A$ and $B$ having $k n$ elements each, for some constant $k$ depending on the problem. We then show that the set $\left\{g \mid f_{G}(g) \leq \varepsilon\right\}$ has $\Omega\left((k n)^{l}\right)=\Omega\left(n^{l}\right)$ complexity, for some constant $l$ depending on the problem. We do this by showing that this set has $\Omega\left(n^{l}\right)$ distinct connected components. Since each one must contain some local minimum of $f_{G}(g)$, this shows that there are $\Omega\left(n^{l}\right)$ local minima in the graph of $f_{G}(g)$. In some cases, we also show that the graph of $F_{G}(g)$ may have this complexity. Previous constructions, such as those in [5] and [4], have been for the directed Hausdorff distance alone.

The constructions for the undirected Hausdorff distance and the constructions for the directed distance on which they are based may have high complexity in a small space: for a fixed $\varepsilon$, we can make $F_{G}(g)$ have $\Omega\left(n^{l}\right)$ complexity in an arbitrarily small region of transformation space (i.e., this does not depend on just shrinking $\varepsilon$ ). This is motivated by the observations in [2] and [7] that, for some groups $G$, if $F_{G}(g) \leq \varepsilon$, then $g$ must lie in a small region in transformation space. If the undirected Hausdorff distance could have only small complexity in a small area, we might be able to obtain efficient algorithms: if the global minimum of $F_{G}(g)$ is restricted to lie in a small area, then searching only this 
Table 1. Lower bounds for the complexity of the Hausdorff distance between two sets of size $n$. The only previously known lower bounds were those by Chew and Kedem [4] for the directed distance under translation (for both sets of points, and sets of points and line segments).

\begin{tabular}{|c|c|c|c|c|c|}
\hline \multirow[b]{2}{*}{ Transformation group } & \multicolumn{3}{|c|}{$\begin{array}{c}\text { Point sets } \\
\text { results for } f_{G}(t) \text { and } F_{G}(t) \\
\text { except } \ddagger \text { for } f_{G}(t) \text { only }\end{array}$} & \multicolumn{2}{|c|}{$\begin{array}{l}\text { Points and segments } \\
\text { results for } f_{G}(t) \text { only }\end{array}$} \\
\hline & $L_{1}$ & $L_{2}$ & $L_{\infty}$ & Any $L_{p}$ & $L_{2}$ \\
\hline $\begin{array}{l}\text { Translation } \\
\text { Rigid motion }\end{array}$ & $\Omega\left(n^{3}\right)$ & $\begin{array}{l}\Omega\left(n^{3}\right) \\
\Omega\left(n^{5}\right)\end{array}$ & $\Omega\left(n^{3}\right)$ & $\Omega\left(n^{4}\right)$ & $\Omega\left(n^{6}\right)$ \\
\hline $\begin{array}{l}\text { Translation and } x, y \text { scale } \\
\text { Affine transformation }\end{array}$ & $\begin{array}{l}\Omega\left(n^{7}\right) \\
\Omega\left(n^{9}\right)\end{array}$ & $\begin{array}{l}\Omega\left(n^{7}\right) \\
\Omega\left(n^{9}\right)\end{array}$ & $\begin{array}{l}\Omega\left(n^{7}\right)^{\ddagger} \\
\Omega\left(n^{9}\right)\end{array}$ & $\begin{array}{l}\Omega\left(n^{8}\right) \\
\Omega\left(n^{12}\right)\end{array}$ & \\
\hline
\end{tabular}

area of its graph can take less time than searching the entire graph. The constructions here show that, in some cases, this is not possible.

In this paper we deal with four transformation groups: the group $T r$ of translations, the group Rig of rigid motions (translations and rotations), the group $S c$ of translations and $(x, y)$ scaling, and the group Aff of nondegenerate affine transformations. Table 1 shows the problems for which we present lower bounds; Table 2 shows the running times of the algorithms which solve those problems. It can be seen that in most cases, the running times are nearly tight with the lower bounds. The exception is the bound for point sets under translation with the $L_{1}$ and $L_{\infty}$ norms in [4], where an algorithm was given which uses the structure of the problem under these norms to avoid explicitly searching the entire graph. It may be possible to develop algorithms using similar techniques for some of the other problems, and so it should be emphasized that the lower bounds presented here are for the complexity of the graph of the Hausdorff distance, and do not necessarily give lower bounds for algorithms that determine the optimal transformation.

\section{Point Sets Under Translation}

Let $t$ be a translation. Define

$$
\begin{aligned}
& F_{T_{r}}(t)=H(A, B \oplus t), \\
& f_{r_{r}}(t)=h(B \oplus t, A)
\end{aligned}
$$

as the (undirected and directed, respectively) Hausdorff distance between $A$ and $B$ as a function of translation. This section describes two constructions of point sets $A$ and

Table 2: Time bounds for algorithms that find the exact minimum Hausdorff distance under transformation between two sets of size $n$.

\begin{tabular}{lccccc}
\hline & \multicolumn{2}{c}{ Point sets } & & \multicolumn{2}{c}{ Points and segments } \\
\cline { 2 - 3 } \cline { 5 - 6 } \multicolumn{1}{c}{ Problem } & $L_{1}, L_{\infty}$ & $L_{2}$ & & $L_{1}, L_{\infty}$ & $L_{2}$ \\
\hline Translation & $O\left(n^{2} \log ^{2} n\right)[4]$ & $\begin{array}{c}O\left(n^{3} \log n\right)[5] \\
O\left(n^{5} \log 2 n\right)[3]\end{array}$ & & $O\left(n^{4} \alpha(n)\right)[5]$ & $O\left(n^{4} \log ^{3} n\right)[1]$ \\
Rigid motion & & & & $O\left(n^{6} \log ^{2} n\right)[3]$ \\
\hline
\end{tabular}


$B$. each containing $O(n)$ points, for which $F_{T r}(t)$ and $f_{T r}(t)$ have $\Omega\left(n^{3}\right)$ local minima within an arbitrarily small area. The first construction is for the $L_{1}$ or $L_{\infty}$ norm; the second is for the $L_{2}$ norm.

\subsection{The $L_{1}$ and $L_{\infty}$ Example}

We use the $L_{\infty}$ norm throughout; rotating the point sets by $45^{\circ}$ gives the construction for $L_{1}$.

Let $A$ consist of two diagonal rows, each of $n$ points spaced $\sigma$ apart (i.e.. $\sigma$ apart in both $x$ and $y$ ). The rows are $2 \varepsilon+\delta$ apart, where $\delta<\sigma / n$. $A$ and $A^{\varepsilon}$ are shown in Fig. 1 . The area left uncovered by $A^{\varepsilon}$ contains a staircase of $\Omega(n)$ steps, in the gap between the two sides. The width of this gap is $\delta$. Note that by reducing $\sigma$, the two rows can be compressed inward, thereby making the stairsteps (and thus the total length of the staircase) as small as desired.

Let $B$ consist of two diagonal rows of points, each of $n$ points, as shown in Fig. 2. The points in each row are slightly more than $\delta$ apart, and are placed so that one row lies around the horizontal part of a stairstep, and the other lies around the adjacent vertical

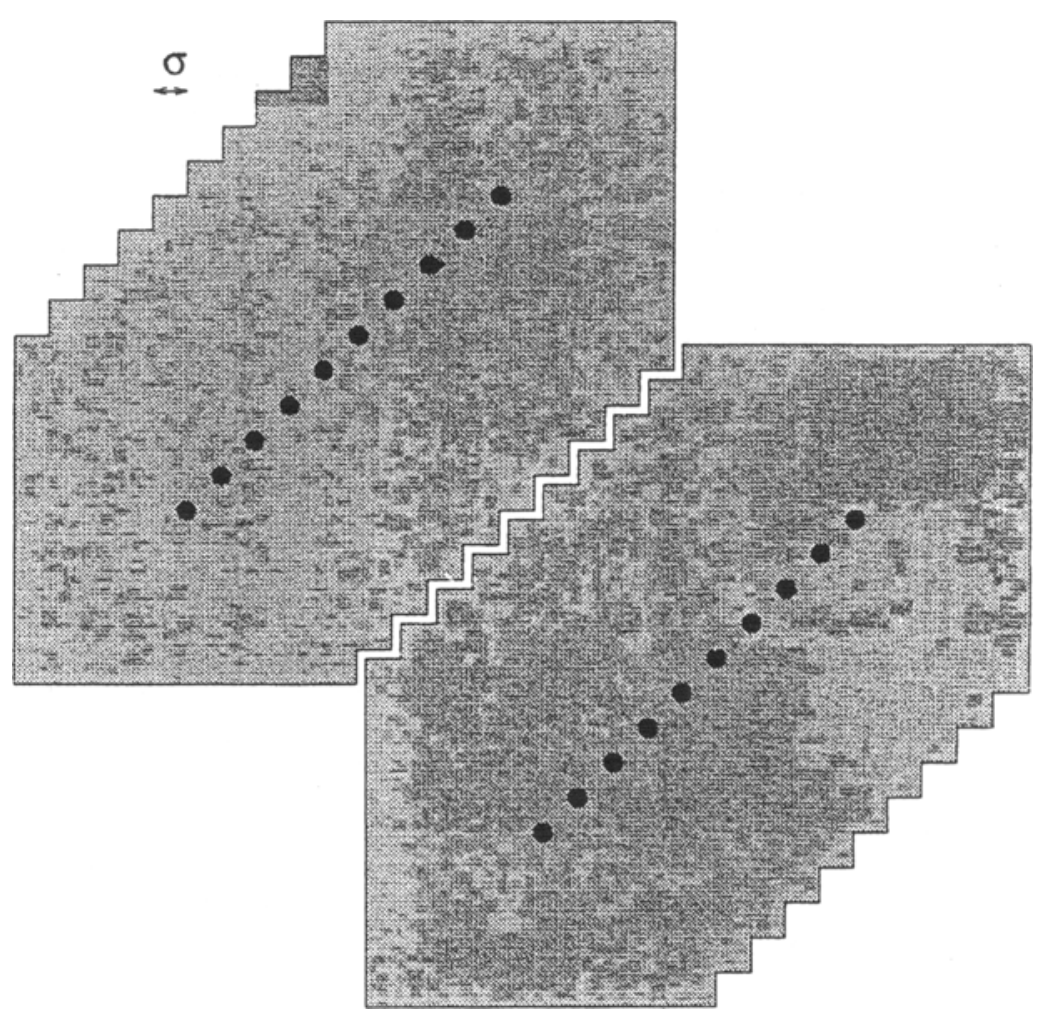

Fig. 1. The sets $A$ and $A^{\varepsilon}$ for the $L_{\infty}$ lower bound for point sets under translation. 


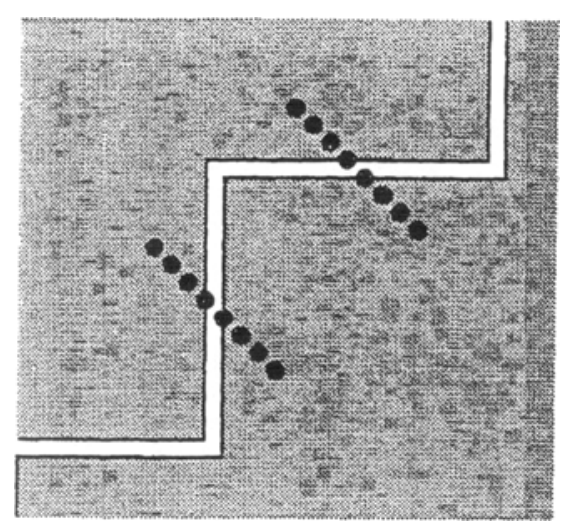

Fig. 2. The sets $A^{\varepsilon}$ and $B$ for the $L_{\infty}$ lower bound for point sets under translation.

part. Since the stairstep lengths are all $\sigma$, and $\delta<\sigma / n$, each row of $B$ is shorter (in height and width) than a stairstep segment.

Consider translating $B$ slightly upward or downward. The points around the vertical stairstep remain inside $A^{\varepsilon}$ or outside it, as they were before, but the points around the horizontal stairstep move into and out of $A^{\varepsilon}$ as $B$ moves. Similarly, as $B$ moves left or right, the points around the vertical stairstep move in and out of $A^{\varepsilon}$, but the points around the horizontal stairstep do not. We can thus see $\Omega\left(n^{2}\right)$ different configurations of $B$ with respect to this one stairstep, since we can independently choose where the gaps lie in the two rows of $B . B$ can also be translated so that it straddles any of the other stairsteps, each of which gives rise to $\Omega\left(n^{2}\right)$ configurations, for a total of $\Omega\left(n^{3}\right)$ configurations. We only consider configurations where there is at least one point of each row of $B$ on either side of the gap. Each one of these configurations can be labeled with three numbers from 1 to $n-1$ : the number of points in the bottom row of $B$ that are inside the upper-left component of $A^{\varepsilon}$ (i.e., to the left of the gap), the number of points in the top row of $B$ that are to the left of the gap, and the number of the stairstep which is straddled by $B . \Omega\left(n^{3}\right)$ such labels are possible. Suppose $t_{1}$ and $t_{2}$ are translations representing configurations with distinct labels. Then $f_{T_{r}}\left(t_{1}\right) \leq \varepsilon$ and $f_{T_{r}}\left(t_{2}\right) \leq \varepsilon$, since each translation places $B$ entirely inside $A^{\varepsilon}$, but any path from $t_{1}$ to $t_{2}$ must pass through a translation $t$ where $f_{T r}(t)>\varepsilon$ : either some point in one of the rows of $B$ must cross the gap, in which case $f_{T r}(t)>\varepsilon$ when $t$ is a translation placing that point inside the gap, or $B$ must be moved so as to straddle another stairstep, in which case again at least one point of $B$ must move through the gap. All these labels therefore label distinct regions.

Another way to visualize this is similar to that used in [4]: define $S(A, \varepsilon, b)$ for some $b \in B$ to be $A^{\varepsilon} \oplus-b$. Then $t \in S(A, \varepsilon, b)$ exactly when $b+t \in A^{\varepsilon}$. This set is therefore the set of all translations which map $b$ into $A^{\varepsilon}$. Now define $S(A, \varepsilon, B)=\bigcap_{b \in B} S(A, \varepsilon, b)$. Then $t \in S(A, \varepsilon, B)$ iff $B \oplus t \subseteq A^{\varepsilon}$, or $f_{T r}(t) \leq \varepsilon ; S(A, \varepsilon, B)$ is therefore the set of all translations $t$ which make $h(B \oplus t, A) \leq \varepsilon$.

We can construct $S(A, \varepsilon, B)$ by making a copy of $A^{\varepsilon}$ for every point in $B$, translating these copies and forming their intersection. Alternately, we can consider making a copy of the complement of $A^{\varepsilon}$ for every point in $B$, translating these copies, and forming their 


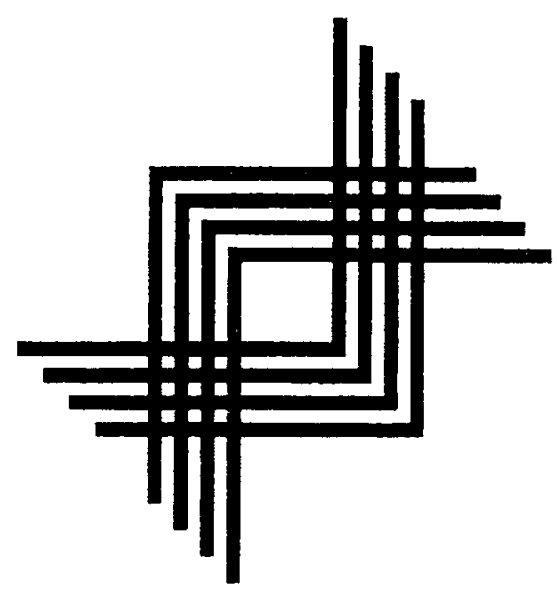

Fig. 3. Part of $S(A, \varepsilon, B)$ for the $L_{\infty}$ lower bound for point sets under translation.

union. This union has a hole for every connected component of $S(A, \varepsilon, B)$. Figure 3 shows part of such a union. Each jagged line represents one segment of the gap staircase of some translation of $A^{\varepsilon}$ (i.e., portions of one copy of the complement of $A^{\varepsilon}$ ). There are two sets of translations of this staircase, corresponding to the two rows of $B$. These two sets intersect in $\Omega(n)$ crosshatches, each having $\Omega\left(n^{2}\right)$ holes; Fig. 3 shows two of the crosshatches.

We now note that $\delta$ can be made as small as desired, thereby narrowing the staircase gap and reducing the lengths of the rows of $B$; and the staircase itself can be compressed as much as is desired by reducing $\sigma$ (as long as $\delta$ stays smaller than $\sigma / n$ ). This means that, for a fixed $n$ and $\varepsilon$, we can compress the $\Omega\left(n^{3}\right)$-complexity region down into an arbitrarily small area, bounded by a square $n \sigma$ on each side, since that is the length of the staircase.

The area where the undirected Hausdorff distance $F_{T r}(t)$ is no greater than $\varepsilon$ can also have large complexity in a small space. Set $\sigma<\varepsilon / n$ so that the rows of $A$ have length less than $\varepsilon$, and add two points to $B$, one in the middle of each row of $A$. Then if the main body of $B$ is translated anywhere on the staircase, these two extra points remain close to the rows of $A$. Since the rows have length less than $\varepsilon$, there is always some point of $B$ within $\varepsilon$ of any point of $A$, for any translation in the complex region. Thus. $H(A, B \oplus t)>\varepsilon$ exactly where $h(B \oplus t, A)>\varepsilon$ (at least in this region of interest), since $h(A, B \oplus t)$ is always at most $\varepsilon$. The undirected Hausdorff distance $F_{T r}(t)$ therefore has complexity $\Omega\left(n^{3}\right)$.

\subsection{The $L_{2}$ Example}

In this subsection we show how the previous example can be modified so that it works with the $L_{2}$ norm. The set $A$ consists of two vertical rows of $n$ points, spaced $\sigma$ apart; the two rows are staggered by $\sigma / 2$ (see Fig. 4). The distance between the rows is set such 


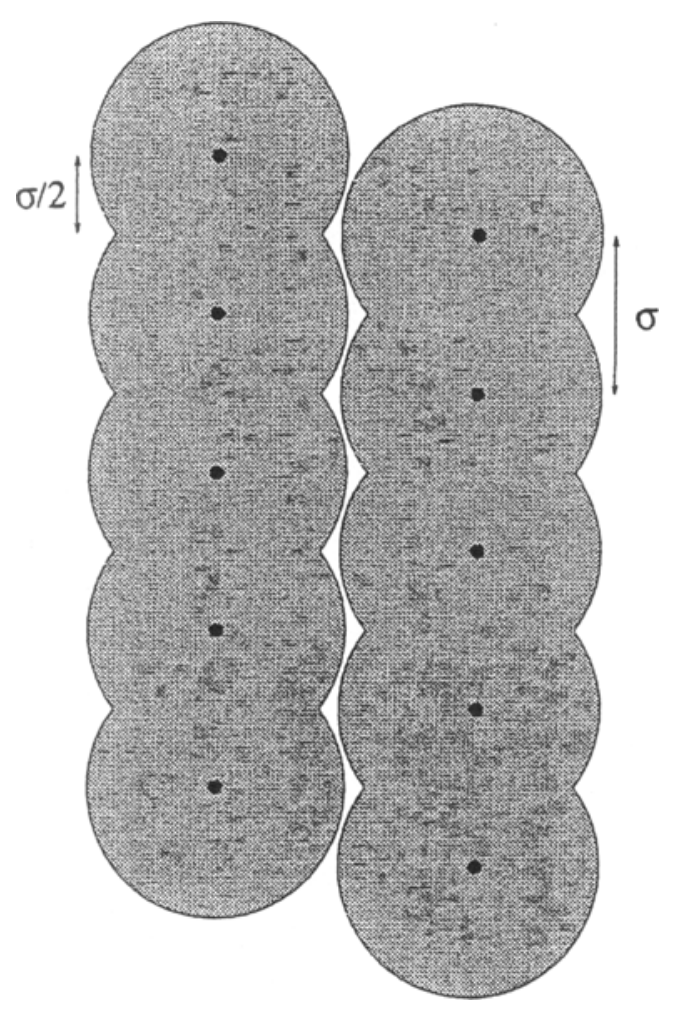

Fig. 4. The sets $A$ and $A^{c}$ for the $L_{2}$ lower bound for point sets under translation.

that the circles of $A^{\varepsilon}$ are $\delta$ apart at their closest approach; the gap between the left and right sides is not of constant width.

The set $B$ again consists of two rows of $n$ points. These rows are horizontal, and spaced $\sigma / 2$ apart. The points in each row are slightly more than $2 \delta$ apart. They are shown superimposed on $A^{\varepsilon}$ in Fig. 5. The idea is that, no matter what values $\sigma, \varepsilon$, and $n$ have, if $\delta$ is small enough, then it is possible to choose $n_{1}$ and $n_{2}$ independently, and position $B$ such that $B \subseteq A^{\varepsilon}$, there are $n_{1}$ points of the top row on the left side of the gap, and $n_{2}$ points of the bottom row on the left side of the gap. This gives $\Omega\left(n^{2}\right)$ possible configurations of $B$ around a single "wobble" in the gap; as there are $\Omega(n)$ such wobbles, there are $\Omega\left(n^{3}\right)$ different configurations of $B$ with $B \subseteq A^{\varepsilon}$. A labeling argument, similar to that in the previous subsection, shows that these configurations are all distinct.

This is difficult to visualize, so again we look at $S(A, \varepsilon, B)$. We construct this, as before, by taking the union of $O(n)$ copies of the gap, translated by various amounts, and showing that this union has $\Omega\left(n^{3}\right)$ disjoint holes.

Since the actual gap has such a complicated shape, we deal only with a small part of it. In particular, we consider only the regions where the gap's width is between $\delta$ and $2 \delta$ (recall that $\delta$ is the width of the narrowest part of the gap). There are $\Omega(n)$ regions where this is true, each one centered around a place where the gap is at its narrowest. We bound each such region by a rectangle. These rectangles are $2 \delta$ wide by $\lambda$ long, where 


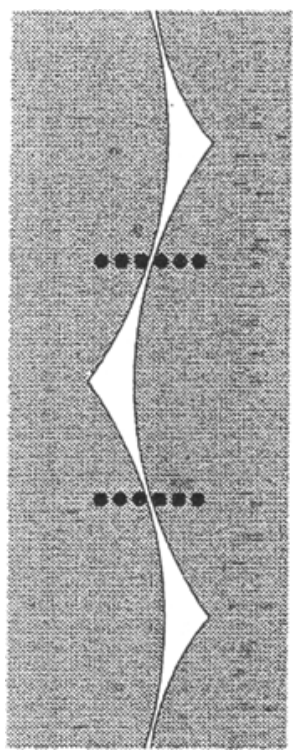

Fig. 5. The sets $A^{\varepsilon}$ and $B$ for the $L_{2}$ lower bound for point sets under translation.

$\lambda$ is determined by $\varepsilon$ and $\delta$, and is equal to $\sqrt{4 \varepsilon \delta-\delta^{2}}$. See Fig. 6 for an illustration of this region. Now, note that $\lambda / \delta=\sqrt{4 \varepsilon / \delta-1}$. Thus, for any fixed $\varepsilon$, we can make $\lambda / \delta$ as large as we like by making $\delta$ small enough: as $\delta$ decreases, the rectangles get both narrower and shorter, but their length-to-width ratio increases.

The gap is narrowest exactly where a line from one point in the left row of $A$ to one of its neighbors in the right row crosses it. The interesting rectangles are oriented perpendicular to such lines. There are two sets of such rectangles, one leaning to the left and the other leaning to the right. The angle between these two sets decreases as $\sigma$ decreases, but is not significantly affected by $\delta$. Suppose that we take $n$ right-leaning rectangles, and position them slightly more than $2 \delta$ apart (the same spacing as the points of $B$ ), so that the right edge of one rectangle almost touches the left edge of the next. Then, for a small enough value of $\delta, \lambda / \delta$ is large enough that one left-leaning rectangle can intersect all $n$ of these right-leaning rectangles.

In constructing $S(A, \varepsilon, B)$, we make $n$ copies of the gap stacked slightly more than $2 \delta$ apart (corresponding to one of the rows of $B$ ), and have these intersect with another $n$ copies, shifted down by $\sigma / 2$ (corresponding to the other row), giving $\Omega\left(n^{3}\right)$ intersections: each left-leaning rectangle from one of the copies intersects $n$ right-leaning rectangles from other copies, and vice versa. Figure 7 shows part of such an arrangement. There are $\Omega\left(n^{2}\right)$ holes in the crosshatch, and $\Omega(n)$ such crosshatches in the complete arrangement. As in Section 2.1, we can independently choose $n_{1}$ and $n_{2}$, and position $B$ in such a way that $n_{1}$ points from its top row lie in the left half of $A^{\varepsilon}$, and $n_{2}$ points from its bottom row lie in the left half. There are $\Omega(n)$ such placements for any $n_{1}$ and $n_{2}\left(1 \leq n_{1}, n_{2}<n\right)$, one around each wobble of the gap, for $\Omega\left(n^{3}\right)$ different configurations. Going from some configuration to another with a different $n_{1}$ or $n_{2}$ involves some point crossing the gap. 


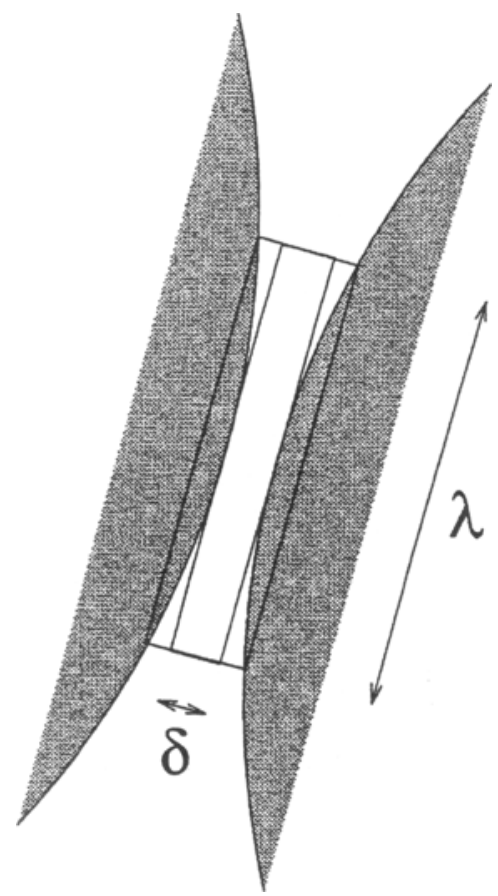

Fig. 6. A closeup of the interesting region of the gap.

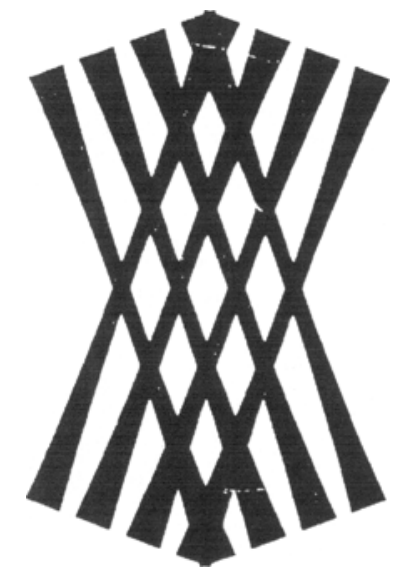

Fig. 7. Part of $S(A, \varepsilon, B)$ for the $L_{2}$ lower bound for point sets under translation. 
Also, since the points of $B$ are spaced about $2 \delta$ apart and the gap becomes wider than this between two adjacent wobbles, it is impossible to translate $B$ from one configuration to another with the same $n_{1}$ and $n_{2}$ without some point moving outside $A^{\varepsilon}$. Thus, all these $\Omega\left(n^{3}\right)$ configurations all belong to different connected components of $S(A, \varepsilon, B)$. These components are all contained in an area which is $O(n \sigma)$ high by $O(n \delta)$ wide. and so by a suitable choice of $\sigma$, this region of high complexity can be made arbitrarily small.

As in Section 2.1, if $n \sigma<\varepsilon$, we may augment $B$ by two points, one each in the middle of the two rows of $A$, such that $h(A, B \oplus t) \leq \varepsilon$ for all translations in the complex region; this construction therefore similarly shows that the undirected Hausdorff distance can have large complexity in a small area.

\section{Sets of Points and Line Segments Under Translation}

This section describes a construction of two sets $A$ and $B$, each consisting of $2 n$ points and nonintersecting line segments, for which the graph of the directed Hausdorff distance as a function of translation $f_{T r}(t)=h(B \oplus t, A)$ has $\Omega\left(n^{4}\right)$ complexity.

Fix $\varepsilon$ and $n$ and pick $\delta$ such that $\delta<\varepsilon / n$. Now let $A$ consist of a group of $n$ horizontal segments, each of length $(n-1)(2 \varepsilon+\delta)$, spaced $2 \varepsilon+\delta$ apart, plus a similar group of $n$ vertical segments. Under any $L_{p}$ norm, $A^{\varepsilon}$ then consists of $n$ horizontal bars and $n$ vertical bars, with gaps of width $\delta$ between adjacent bars; the shape of the caps on the ends of the bars depends on the exact norm. Now, let $B$ consist of a vertical row of $n$ points, spaced $2 \delta$ apart, located at the bottom-left comer of the group of horizontal lines in $A$, plus a similar horizontal row located at the bottom-left corner of the group of vertical lines. Figure 8 shows $B$ overlaid on $A$ and $A^{\varepsilon}$.

There are $\Omega\left(n^{4}\right)$ different configurations of $B$ with respect to $A$ : the vertical row of $B$ can be straddling any of the $n-1$ gaps, and from 1 to $n-1$ points can lie below the gap; similarly, the horizontal row can be placed in any one of $\Omega\left(n^{2}\right)$ different
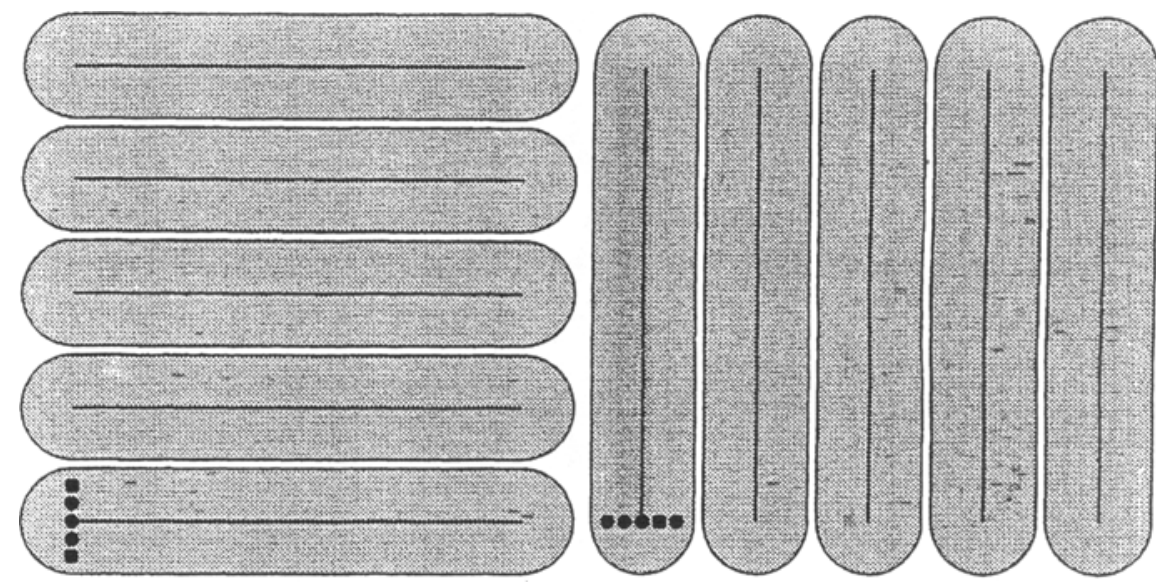

Fig. 8. The sets $A, A^{\varepsilon}$, and $B$ for points and segments under translation. 
configurations with respect to the vertical segments of $A$. Sliding $B$ horizontally does not affect the configuration of the vertical row, and sliding it vertically does not affect the configuration of the horizontal row (as long as these rows remain within limits); the configurations of the two rows may thus be chosen independently, for a total of $\Omega\left(n^{4}\right)$ different configurations. These are all clearly distinct, since any two differ in the number of points of $B$ contained in one of the connected components of $A^{\varepsilon}$.

\section{Point Sets Under Rigid Motion}

Let $t$ be a translation and let $\theta$ be an angle. Define

$$
\begin{aligned}
F_{R i g}(t, \theta) & =H\left(A, r_{\theta}(B) \oplus t\right), \\
f_{\text {Rig }}(t, \theta) & =h\left(r_{\theta}(B) \oplus t, A\right),
\end{aligned}
$$

where $r_{\theta}(B)$ denotes the set obtained by rotating $B$ by $\theta$ counterclockwise about the origin. We use the $L_{2}$ norm wherever we deal with rotation, since it is the only rotationally symmetric $L_{p}$ norm.

The following construction shows that there can be $\Omega\left(n^{5}\right)$ distinct connected components in $(t, \theta)$ space where the undirected Hausdorff distance $F_{\text {Rig }}(t, \theta)$ between two sets of $\Omega(n)$ points is less than $\varepsilon$. It is based on an augmentation of $A$ and $B$ from Section 2.2. For clarity, we refer to the sets $A$ and $B$ constructed for the translational lower bounds as $A_{T r}$ and $B_{T r}$.

First, note that it is possible to rotate $B_{T r}$ from that construction by some very small angle $\theta_{\min }$ about its centroid while still maintaining the $\Omega\left(n^{3}\right)$ complexity of $h\left(B_{\operatorname{Tr}} \oplus\right.$ $\left.t, A_{T r}\right)$. This is because there must be, in the $\Omega\left(n^{3}\right)$ arrangement of connected components of $S(A, \varepsilon, B)$, some minimum distance between features, and so any rotation that does not move any feature of the arrangement more than half this distance cannot change the overall topology of the arrangement: none of the connected components merge, nor do any vanish.

The augmentation to $A_{T r}$ consists of $n$ points along a vertical line, spaced less than $\varepsilon /(2 n)$ apart. Placing a disk of radius $\varepsilon$ about each gives a shape with two vertical scalloped edges: the left and right sides are close to vertical, but have $n$ slight bulges or lobes. Call this row of points $A_{2}$.

Now, if $A_{2}$ is located sufficiently far from the origin, and perpendicular to the line joining it to the origin, then it is possible to pass a circular arc (centered at the origin) through the inner scalloped edge so that it passes through each of the $n$ lobes and the gaps between them. The arc does not pass through these lobes evenly, but cuts deeper into some of them than others. However, by moving the row farther away and thus increasing the radius of the arc, we may control the magnitude of this effect, since the arc approaches a straight line as $A_{2}$ moves away. By slightly adjusting the radius of the arc, we may also control the ratio between the arc length contained inside the lobes and the arc length contained in the spaces between the lobes. We place $A_{2}$ far enough away and position the circular arc such that the ratio between the arc length contained in any lobe and the arc length contained in the space next to that lobe is greater than $8 n: 1$. Let the shortest of the arc lengths contained in the lobes be $l$ (see Fig. 9). As the arc becomes closer to 


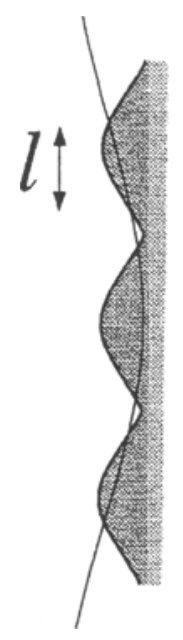

Fig. 9. The interaction between $A_{2}^{\boldsymbol{c}}$ and $B_{2}$.

a straight line (as $A_{2}$ is moved away from the origin), the lengths of the arc segments contained in the lobes become more similar (and, at the limit, are all equal). $A_{2}$ should be positioned far enough away that they are all within a factor of two of each other. The widest gap is then smaller than $l /(4 n)$.

We now construct $B_{2}$, which consists of $n$ points positioned $l /(2 n)$ apart along this circular arc, initially located in the lowest lobe of $A_{2}^{\varepsilon}$. We also add an extra point to $B_{2}$; this point is initially located at the lower end of $A_{2}$. Now, as $B_{2}$ rotates about the origin, this row of points moves along this circular arc. Since the spaces between lobes along this path are all less than $l /(4 n)$ across, and the entire arc of points fits into a single lobe, only one point passes through a gap at a time, and there are $\Omega\left(n^{2}\right)$ different configurations of $B_{2}$ with respect to this part of $A_{2}^{\varepsilon}$ : there are $n-1$ gaps to be straddled. and, for each gap, between 1 and $n-1$ points of $B_{2}$ can be above the gap. Note that all of these configurations have the property that all of the points of $A_{2}$ are within $\varepsilon$ of some point of $B_{2}$, specifically the extra point.

Pick $\theta$, the amount by which $B_{2}$ is rotated about the origin, such that the points of $B_{2}$ are straddling one of the spaces between lobes, and such that this straddling is even: the two points closest to the gap are equal distances away from the edges of the gap. They are at least $l /(8 n)$ away from these edges. Now, consider translating the points of $B_{2}$ vertically up or down by up to $l /(16 n)$. If the arc along which the points lie is close enough to a straight vertical line, then they will stay inside $A_{2}^{\varepsilon}$. Let $d$ be the minimum horizontal "depth" inside $A_{2}^{E}$ achieved for any point of $B_{2}$ at any point in this translational range. Figure 10 shows this situation; the vertical bars are $l /(8 n)$ high $(l / 16 n$ above and below the center). Let $d_{\min }$ be the smallest such $d$ value achieved for any of the $\Omega\left(n^{2}\right)$ possible such straddling configurations. Also, let $w$ be the minimum distance between the circular arc and the bottom of the space between two lobes.

We now construct $A_{T r}$ and $B_{T r}$ as in Section 2.2 by choosing $\sigma$ and $\delta$ so that the $\Omega\left(n^{3}\right)$ complexity region of $F_{T r}(t)$ is at most $l /(16 n)$ high by $\min \left(d_{\min } / 2, w / 2\right)$ wide, and so that the centroid of $B_{T r}$ is at the origin. Let $A=A_{T r} \cup A_{2}$ and $B=B_{T r} \cup B_{2}$. 


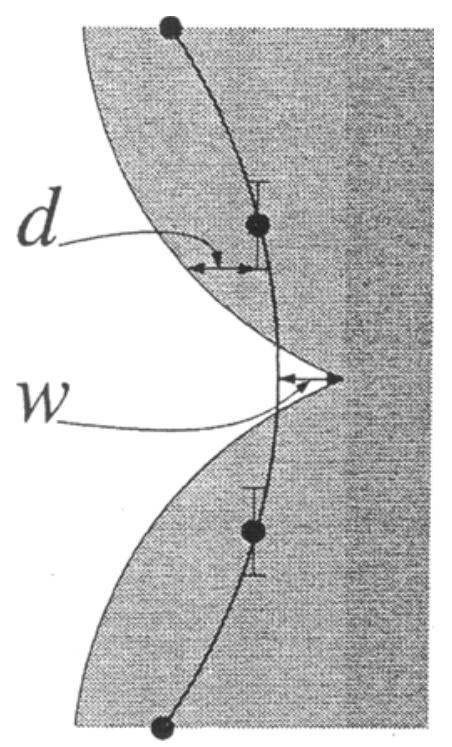

Fig. 10. The minimum depth of the points of $B_{2}$.

Let $\theta$ produce one of the straddling configurations described above. Then, if $|\theta|<\theta_{\min }$, there are $\Omega\left(n^{3}\right)$ different connected components in $t$ space where $F_{R i g}(t, \theta) \leq \varepsilon$. This is because a slight translation of $B_{2}$ with respect to $A_{2}$ does not move any of the points of $B_{2}$ out of $A_{2}^{\varepsilon}$, and $B_{T r}$ and $A_{T r}$ have been constructed so that the range of translations required is very small. Note that $F_{R i g}(t, \theta)$ is determined by $f_{R i g}(t, \theta)$ for all transformations in the range under consideration, as the directed distance from $A$ to the rotated and translated $B$ is always less than $\varepsilon$.

A labeling argument similar to that in Section 2.1 now shows that there are $\Omega\left(n^{5}\right)$ different connected components in $(t, \theta)$ space where $F_{R i g}(t, \theta) \leq \varepsilon, \Omega\left(n^{3}\right)$ corresponding to each of the $\Omega\left(n^{2}\right)$ such values of $\theta$. A key point in the argument is that it is not possible for one of the points of $B_{2}$ to sneak around the space between the lobes (through the main body of $A_{2}^{\varepsilon}$ ), since it would have to translate at least $w$ away from the original circular arc, which would force at least one point of $B_{T r}$ to cross some gap. Thus, any two configurations which differ in how the points of $B_{2}$ are straddling the gaps of $A_{2}^{\varepsilon}$ must belong to different connected components.

There is a problem with this construction as it has been presented: $A_{2}$ must subtend an angle of less than $\theta_{\min }$, which depends on $\sigma$ and $\delta$, which depend on $l, d_{\min }$, and $w$, which depend on the circular arc along which $B_{2}$ is placed, which must have a larger radius for a smaller $\theta_{\min }$ and thus depends on $\theta_{\min }$; the parameters are thus interdependent. However, as $A_{2}$ and $B_{2}$ are moved farther out, $l, d_{\mathrm{min}}$, and $w$ approach limit values, as the circular arc becomes closer to a straight line. Thus, we can initially place $A_{2}$ and $B_{2}$ where $l$ and $w$ are within some small factor (say, within 1\%) of their limit values, then determine $\sigma$ and $\delta$ which work for any values of $l$ and $w$ between their original values and their limit values, and thus determine $\theta_{\min }$. This gives us a minimum value for the radius of the circular arc, and we know that we can move $A_{2}$ and $B_{2}$ out farther if necessary without affecting the validity of the construction. 

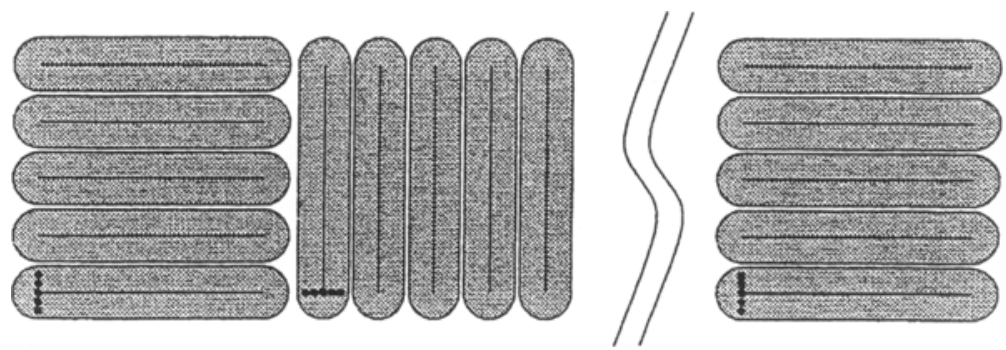

Fig. 11. The sets $A, A^{\varepsilon}$, and $B$ for points and segments under rigid motion.

\section{Sets of Points and Line Segments Under Rigid Motion}

This example is a modification of the example from Section 3, using the techniques from Section 4. Again, we refer to the sets $A$ and $B$ constructed in Section 3 as $A_{T r}$ and $B_{T r}$. As before, we observe that the set $B_{T r}$ may be rotated by some small angle $\theta_{\min }$ about its centroid without changing the topology of the arrangement.

Place $B_{T_{r}}$ so that its centroid is at the origin (the center of rotation). Augment $A_{T r}$ by a group of segments $A_{2}$ identical to the left-hand group of $A_{T r} . A_{2}$ is placed so that it subtends a total angle of less than $\theta_{\min }$ to the origin, and lies directly to the right of it. Let $A=A_{T r} \cup A_{2}$. We also add $n$ points to $B_{T r}$ in a vertical row, in the same relative position to $A_{2}$ as the first vertical row of $B_{T r}$ was to the left-hand group of $A$. Call this new row $B_{2}$ and let $B=B_{T_{r}} \cup B_{2} . A, A^{\varepsilon}$, and $B$ are shown in Fig. 11.

Now, any translation $t$ for which $B_{T r} \oplus t \in A_{T r}^{\varepsilon}$ also has $B \oplus t \in A^{\varepsilon}$. Fix such a $t$ and consider values of $\theta$ where $|\theta|<\theta_{\min }$. As $\theta$ changes through this range, the points in $B_{2}$ sweep across the gaps in $A_{2}^{\varepsilon}$. Their spacing is such that only one point crosses a gap at a time. Thus, as we vary $\theta$, the points of $B_{2}$ achieve $\Omega\left(n^{2}\right)$ different configurations with respect to the gaps of $A_{2}^{\varepsilon}$. For this choice of $t$, there are thus $\Omega\left(n^{2}\right)$ values of $\theta$ for which $f_{\text {Rig }}(t, \theta) \leq \varepsilon$, since any rotation in this range keeps $r_{\theta}(B) \oplus t$ inside $A^{\varepsilon}$. We can choose $t$ to represent one of the $\Omega\left(n^{4}\right)$ distinct configurations of $B$ with respect to $A$, and so this gives $\Omega\left(n^{6}\right)$ different configurations of $B$ with respect to the gaps of $A^{\varepsilon}$ for which $f_{\text {Rig }}(t, \theta) \leq \varepsilon$. These configurations are not connected in $(t, \theta)$ space, since any path from one to another must cause at least one point to cross some gap.

\section{Point Sets Under Translation and Scale}

In this section we consider the complexity of the graph of the Hausdorff distance between two sets of points as one set is translated and scaled with respect to the other. This scaling is with respect to fixed $x$ and $y$ axes. This transformation group is of interest because it has been used in image-recognition tasks, as described in [6]. It corresponds to the transformations that the image of an object may undergo as a camera is moved forward or panned about a vertical axis, under the weak perspective projection model.

We present three different lower-bound constructions: one each for the $L_{1}, L_{2}$, and $L_{\infty}$ norms. They are all similar in concept, and are based on the constructions in Section 2 , 
using techniques from Section 4. The $L_{1}$ and $L_{2}$ constructions show lower bounds for both the directed and undirected Hausdorff distance; the $L_{\infty}$ construction, however, shows a lower bound for the directed Hausdorff distance only.

Define the undirected and directed Hausdorff distance as functions of a translation $t$ and two scale parameters $s_{x}$ and $s_{y}$ as

$$
\begin{aligned}
& F_{S c}\left(t, s_{x}, s_{y}\right)=H\left(A, S_{s_{x} s_{y}}(B) \oplus t\right), \\
& f_{S_{c}}\left(t, s_{x}, s_{y}\right)=h\left(S_{s_{x} s_{y}}(B) \oplus t, A\right),
\end{aligned}
$$

where $S_{s_{x} s_{y}}(B)$ denotes the set $\left\{\left(s_{x} x, s_{y} y\right) \mid(x, y) \in B\right\}$ (i.e., the set obtained by scaling $B$ by a factor of $s_{x}$ in the $x$ direction and $s_{y}$ in the $y$ direction).

The central idea behind these constructions is that changing $s_{x}$ slightly has very little effect on points which are near the $y$ axis, while it has a largely translational effect on groups of points which are located a large distance along the $x$ axis ("large" here means that the distance of the group from the origin is large relative to the size of the group).

\subsection{The $L_{2}$ Example}

This example is very similar to the example for point sets under rigid motion. Again, a key observation is that the construction of $A_{T r}$ and $B_{T r}$ in Section 2.2 can be perturbed slightly without affecting the topology of the graph. In this case, this perturbation takes the form of a slight scaling of $B_{T r}$ in $x$ and $y$. Suppose that the origin is placed at the lower-left corner of $B_{T r}$. Then let $s_{\min }$ be the valid range of such scaling: if $1-s_{\min } \leq s_{z}, s_{y} \leq 1+s_{\min }$, then there are $\Omega\left(n^{3}\right)$ translational configurations of $B_{T r}$ with respect to $A_{T_{r}}$ for which replacing $B_{T_{r}}$ by $S_{s_{x} s_{r}}\left(B_{T_{r}}\right)$ does not change the configuration (i.e., the same points are on the same sides of the gap of $A_{T r}^{\varepsilon}$ ). $s_{\min }$ clearly depends on the $n, \varepsilon$, and $\sigma$ used to construct $A_{T_{r}}$ and $B_{T_{r}}$.

First, we construct $A_{2}$ as in Section 4, and place it so that the $y$ axis cuts through the left-hand scalloped edge in a manner similar to that described in Section 4: each lobe of the scalloped edge contains a length $l$ of the $y$ axis, the smallest depth of any of the gaps is $w$, and the ratio between the axis length contained in any lobe (i.e., $l$ ) and the length contained in the space next to that lobe is greater than $8 n: 1$. Note that this construction does not depend on the location of this copy of $A_{2}$ along the $y$ axis.

We next construct $A_{T r}$ and $B_{T r}$ as in Section 2.2 such that the region of $\Omega\left(n^{3}\right)$ complexity occupies a region less than $\min (w / 2, l /(8 n))$ square, and position them so that the lower-left comer of this region in translation space is located at $t=(0,0)$, and the lower-left corner of $B_{T r}$ is at the origin. This determines a value for $s_{\min }$. We then position the copy of $A_{2}$ a distance of $2 \varepsilon / s_{\min }$ above the origin, with the $y$ axis cutting through it as described above. We also make a copy of $A_{2}$, rotated by $90^{\circ}$, to the right of the copy of $A_{T r}$, with the $x$ axis cutting through the lower scalloped edge in the same manner. $A$ consists of $A_{T r}$ together with these two copies of $A_{2} . B$ then consists of $B_{T r}$ plus two rows of $n$ points plus an extra point per row, as described in Section 4; one row and its extra point are positioned along the $y$ axis inside the uppermost lobe of the first copy of $A_{2}$; the other row and its extra point are in the corresponding position on the $x$ axis, in the second copy of $A_{2}$. Call these two rows $B_{2}$. 
Now, it is possible to choose independently seven numbers $n_{1}, \ldots, n_{7}$ from 1 to $n-1$ and construct a translation $t$ and scale $s_{x}, s_{y}$ of $B$ with respect to $A$ where $f_{s c}\left(t, s_{x}, s_{y}\right) \leq \varepsilon$ in the following manner:

1. $n_{1}, n_{2}$, and $n_{3}$ determine the translation. They are used to position $n_{1}$ of the points of the lower row of $B_{T r}$ to the left of the gap in $A_{T_{r}}^{\varepsilon}, n_{2}$ of the points of the upper row to the left of the gap, and with the rows straddling the $n_{3}$ th wobble of the gap.

2. $n_{4}$ and $n_{5}$ determine $s_{y}$. They are used to position $n_{4}$ of the points of the row of $B_{2}$ lying on the $y$ axis below one of the gaps in the scalloped edge of the upper copy of $A_{2}, n_{5}$ selects the gap. Note that changing $s_{y}$ acts as (essentially) a translation of this row, as its distance from the origin (and therefore its $y$ coordinate) greatly exceeds its length. It is possible to do this no matter what translation was chosen above, since the range of translations is small. Also, any $s_{y}$ chosen in this manner does not exceed the range determined by $s_{\min }$.

3. $n_{6}$ and $n_{7}$ similarly determine $s_{x}$, by positioning the points of the row of $B_{2}$ lying on the $x$ axis with respect to its copy of $A_{2}$.

If two such configurations are generated with different $n_{i}$ values, then it is not possible to move from one to the other without some point crossing a gap, and so they must belong to different connected components in transformation space where $f_{S_{c}}\left(t, s_{x}, s_{y}\right) \leq \varepsilon$. Also, due to the extra points added to the various parts of $B, h\left(A, S_{s_{x} s_{y}}(B) \oplus t\right)$ is no greater than $\varepsilon$ for all such configurations; $F_{S c}$ therefore has $\Omega\left(n^{7}\right)$ distinct local minima. These can occur in an arbitrarily small region of transformation space for a fixed $\varepsilon$.

\subsection{The $L_{1}$ Example}

This example is quite similar to the example in the previous subsection. The construction uses a copy of $A_{T r}$ and $B_{T r}$ from Section 2.1 (the transiational example for $L_{\infty}$ ), rotated $45^{\circ}$. Instead of $A_{T r}$ being augmented by two rows of points, each generating a scalloped edge, it is instead augmented by two rows of points, each generating a sawtooth edge; however, these augmentations are used in the same manner.

\subsection{The $L_{\infty}$ Example}

This example must be constructed differently from the previous two, since a vertical row of points, when dilated by $\varepsilon$, generates a straight vertical edge, with no irregularities that can be exploited. Let $A_{2}$ be a vertical row of $n$ points spaced $2 \varepsilon+\delta$ apart, lying on the $y$ axis, far away from the origin. $A_{2}^{\varepsilon}$ is then a row of $n$ squares with a $\delta$-wide gap between adjacent squares. Let $B_{2}$ be a vertical row of $n$ points spaced just over $\delta$ apart, lying close to $A_{2}$. As $s_{y}$ varies, $B_{2}$ moves mostly translationally, and its points therefore move through the $n-1$ gaps in $A_{2}^{\varepsilon}$, one point at a time. $\Omega\left(n^{2}\right)$ configurations of the points of $B_{2}$ relative to the gaps of $A_{2}^{\varepsilon}$ are therefore possible.

Let $A$ be a copy of $A_{T r}$ from Section 2.1, plus $A_{2}$ as described above, plus a similar horizontal row of $n$ points lying along the $x$ axis. Similarly, let $B$ be a copy of $B_{T r}$ plus $B_{2}$ plus a row of points on the $x$ axis. We first choose a translational configuration 
of $B_{T r}$ with respect to $A_{T r}$, and initially set $s_{x}$ and $s_{y}$ to 1 . We then choose one of the $\Omega\left(n^{2}\right)$ configurations of $B_{2}$ with respect to $A_{2}$, and adjust $s_{y}$ so that this configuration is achieved. We similarly choose one of the $\Omega\left(n^{2}\right)$ configurations of the parts of $A$ and $B$ lying along the $x$ axis, and set $s_{x}$ accordingly. Thus, there are $\Omega\left(n^{7}\right)$ different configurations of $B$ with respect to $A ; f_{S c}(t)$ therefore has $\Omega\left(n^{7}\right)$ distinct local minima.

Unlike the other constructions for point sets, it is not possible to augment $B$ by a few points so that $h(A, t(B))$ is always below $\varepsilon$ for the region of transformation space of interest. This construction is therefore valid for the directed distance only.

\section{Sets of Points and Line Segments Under Translation and Scale}

This example is quite similar to the previous example; we make a copy of $A_{T r}$ and $B_{T r}$ from Section 3, shown in Fig. 8; these copies are placed near the origin, together with a copy of the right-hand group of each (the group exploiting vertical gaps) placed some distance away along the $x$ axis, and a copy of the left-hand group of each placed some distance away along the $y$ axis. This construction gives $\Omega\left(n^{8}\right)$ distinct local minima for $f_{S c}\left(\Omega\left(n^{4}\right)\right.$ from $A_{T r}$ and $B_{T r}$, and $\Omega\left(n^{2}\right)$ from each of the additional groups). As before, the exact norm being used is not relevant, since it does not affect the gaps between the segments.

\section{Point Sets Under Affine Transformation}

Here we are dealing with transformations which map $B$ to $M(B) \oplus t$, where $M$ is a nonsingular $2 \times 2$ matrix defined by

$$
M=\left[\begin{array}{ll}
m_{00} & m_{01} \\
m_{10} & m_{11}
\end{array}\right]
$$

and $t=\left(t_{x}, t_{y}\right)$ is a translation. In other words, each point $\left(b_{x}, b_{y}\right) \in B$ is mapped to $\left(m_{00} b_{x}+m_{01} b_{y}+t_{x}, m_{10} b_{x}+m_{11} b_{y}+t_{y}\right)$. As before, we define

$$
\begin{aligned}
F_{A f f}(t, M) & =H(A, M(B) \oplus t), \\
f_{A f f}(t, M) & =h(M(B) \oplus t, A) .
\end{aligned}
$$

The key observation here is that if $B$ consists of three groups of points, one near the origin, one located a large distance along the $x$ axis, and one located a large distance along the $y$ axis, then:

- Changing $m_{00}$ slightly causes the second group in the transformed $B$ to translate in $x$, but has little other effect.

- Changing $m_{10}$ slightly causes the second group to translate in $y$, but has little other effect.

- Changing $m_{01}$ or $m_{11}$ slightly similarly causes the third group to translate in $x$ or $y$, and has little other effect.

- If $M$ is sufficiently close to the identity matrix, then $M(B)$ is essentially the same as $B$, with the relative positions of the three groups shifted around somewhat. 
The magnitude of these translational motions with respect to their other effects can be increased by moving the corresponding group farther away from the origin along the appropriate axis. Thus, we have a six-parameter system (two translational and four linear parameters), which can be decomposed into three two-parameter translational systems, plus a small amount of slop, which can be made as small as required. In order to build an $\Omega\left(n^{9}\right)$ example, we simply take three copies of the appropriate $A_{T r}$ and $B_{T r}$, and arrange them as described above. Again, we can move copies 2 and 3 of $A_{T r}$ and $B_{T r}$ out from the origin until all nontranslational effects are not significant, since as they move farther out, the amounts by which the $m_{i j}$ values need to be adjusted are reduced. Since all the copies of $A_{T r}$ and $B_{T r}$ have $\Omega\left(n^{3}\right)$ complexity under translation, for both the directed and undirected Hausdorff distance, and are essentially independent, this construction gives $\Omega\left(n^{9}\right)$ complexity for both $f_{A f f}$ and $F_{A f f}$.

\section{Sets of Points and Line Segments Under Affine Transformation}

This example is constructed identically to the previous example. We simply take three copies of $A_{T r}$ and $B_{T r}$ from Section 3 and position one at the origin, one out along the $x$ axis, and one out along the $y$ axis. This gives $\Omega\left(n^{12}\right)$ local minima of $f_{A f f}$, from three essentially independent $\Omega\left(\dot{n}^{4}\right)$ translational examples.

\section{Conclusion}

We have presented constructions which give lower bounds on the complexity of the directed (and, in many cases, the undirected) Hausdorff distance in several different contexts, summarized in Table 1. In a number of of the cases, we have shown that the directed and undirected Hausdorff distances can have large complexity in a small space. The large exponents in these bounds imply that, to solve these problems, either one will have to approximate the Hausdorff distance, or come up with algorithms that can somehow get around them.

We have demonstrated the complexity of the Hausdorff distance as a function of transformation by constructing cases where it has a large number of local minima. A related question is the number of global minima that it can have. In some cases, such as the constructions for point sets under translation with the $L_{1}$ or $L_{\infty}$ norms, it is possible to make the function have equal values at all of the local minima that we have constructed, so that they are all (equal) global minima; in other cases, the number of global minima is an open question.

The problems for which lower bounds on the complexity of the undirected Hausdorff distance were not shown were those involving sets of points and line segments, plus the problem of point sets under translation and scale with the $L_{\infty}$ norm. A remaining.open problem is that of determining bounds for these cases. For example, can the undirected Hausdorff distance under translation between sets of points and segments have any complexity greater than $\Omega\left(n^{3}\right)$ ? Also, is it possible to develop algorithms such as those in [4] which find the minimum Hausdorff distance under the action of some transformation group in less time than that given by the complexity of the graph of the Hausdorff distance function? 


\section{Acknowledgments}

The $\Omega\left(n^{4}\right)$ example shown in Section 3 is due to Paul Chew and Klara Kedem.

\section{References}

1. P. K. Agarwal, M. Sharir, and S. Toledo. Applications of parametric searching in geometric optimization. Proc. Third ACM-SIAM Symposium on Discrete Algorithms, pages 72-82, 1992.

2. H. Alt, B. Behrends, and J. Blömer. Approximate matching of polygonal shapes. Discrete and Computational Geometry, 9:267-291, 1993.

3. L. P. Chew, M. T. Goodrich, D. P. Huttenlocher, K. Kedem, J. M. Kleinberg, and D. Kravets. Geometric partern matching under Euclidean motion. Proc. Fifth Canadian Conference on Computational Geometry, pages 151--156, Waterloo, Ontario, August 1993.

4. L. P. Chew and K. Kedem. Improvements on approximate pattern matching problems. In O. Nurmi and E. Ukkonen, editors, Proc. Third Scandinavian Workshop on Algorithm Theory, pages 318-325. Lecture Notes in Computer Science, Vol. 621, Springer-Verlag, Berlin, 1992.

5. D. P. Huttenlocher, K. Kedem, and M. Sharir. The upper envelope of Voronoi surfaces and its applications. Discrete and Computational Geometry, 9(3):267-291, 1993.

6. D. P. Huttenlocher and W. J. Rucklidge. A multi-resolution technique for comparing images using the Hausdorff distance. Proc. Computer Vision and Pattern Recognition Conference, pages 705-706, New York, 1993.

7. G. Rote. Computing the minimum Hausdorff distance between two point sets on a line under translation. Information Processing Letters, 38(3):123-127, May 1991.

Received August 12, 1994, and in revised form November 5, 1995. 